\title{
The Association of Patient-Reported Local Allergic Symptoms with Postoperative Outcomes of Chronic Rhinosinusitis
}

\author{
Myung Jin Park, $\mathrm{MD}^{1}$, Hye Kyu Min, $\mathrm{MD}^{1}$, Dae Woong Kang, $\mathrm{MD}^{1}$, Hyung Sik Kim, $\mathrm{MD}^{1}$, \\ Su Young Jung, $\mathrm{MD}^{1,2}$, Kun-Hee Lee, $\mathrm{MD}$, $\mathrm{PhD}^{1}$, Sung Wan Kim, $\mathrm{MD}, \mathrm{PhD}^{1}$ and Jin-Young Min, $\mathrm{MD}, \mathrm{PhD}^{1}$ \\ ${ }^{I}$ Department of Otorhinolaryngology-Head and Neck Surgery, School of Medicine, Kyung Hee University, Seoul; and \\ ${ }^{2}$ Department of Otorhinolaryngology-Head and Neck Surgery, Hanyang University Myongji Hospital, Goyang, Korea
}

Background and Objectives: Patients with nonallergic rhinitis (Non-AR) or idiopathic rhinitis are common, with both conditions being classified as local allergic rhinitis (LAR). However, the link between LAR and CRS has not been identified. This study aimed to investigate the association of patient-reported local allergic symptoms with the postoperative outcomes of CRS. Materials and Method: We reviewed the medical records of 64 patients who underwent endoscopic sinus surgery for CRS. All patients underwent skin prick test, Multiple Allergosorbent Test, and computed tomography. Sneezing and nasal itching were defined as local allergic symptoms (LAS). We evaluated the relationships between clinical characteristics and recurrence rate of CRS according to the presence or absence of LAS.

Results: In Non-AR patients, there was no significant difference in age, sex, and TNSS between the LAS (+) and LAS (-) groups. However, in all patients, the CRS recurrence rate was higher in the LAS (+) group (46.7\%) than in the LAS (-) group $(15.8 \% ; \mathrm{p}=0.02)$. A similar trend was observed in the Non-AR patients, showing that the CRS recurrence rate was significantly higher in the LAS $(+)$ group $(56 \%)$ than in the LAS $(-)$ group $(16.7 \%$; $=0.024)$.

Conclusion: The CRS recurrence rate was higher in patients with LAS regardless of the presence of AR.

KEY WORDS: Chronic rhinosinusitis (CRS) - Allergic rhinitis (AR) · Local allergic rhinitis (LAR) · Recurrence rate.

\section{INTRODUCTION}

Chronic rhinosinusitis (CRS) is defined as nasal obstruction, mucopurulent rhinorrhea, postnasal drip, and inflammation of the nasal cavity and sinus that lasts for at least 12 weeks. ${ }^{1)}$ Allergic rhinitis (AR) is known to be one of the risk factors associated with the development of CRS. ${ }^{2) 3}$ It is a series of inflammatory processes that occur within the nasal mucosa and is known to cause CRS by decreasing the integrity of the nasal mucosa and the patency of the sinus, as well as increasing nasal secretions.

$\mathrm{AR}$ and non-allergic rhinitis (Non-AR) are diagnosed on the basis of clinical symptoms as well as the response to an allergic skin prick test (AST) and the presence of serum specific immunoglobulin E antibody (sIgE). Recently, however, concern has been raised regarding varied interpretations of the test, and it has been argued that there is a problem with the existing rhinitis classification scheme. ${ }^{4) 5}$ Local allergic rhinitis (LAR) is diagnosed when such symptoms as rhinorrhea, nasal obstruction, sneezing, itching, and local production of $\operatorname{IgE}$ are observed after exposure to a specific allergen with no evidence of systemic allergies. ${ }^{6-10)}$ LAR is pathophysiologically characterized by the local production of IgE, Th2 cytokine mucosal cell infiltration, the secretion of tryptase and eosinophil cationic protein (ECP), and a positive NAPT result. In previous studies, 47\%

Received: September 4, 2018 / Revised: March 19, 2019 / Accepted: March 19, 2019

Address for correspondence: Jin-Young Min, MD, PhD, Department of Otorhinolaryngology-Head and Neck Surgery, Kyung Hee University, 23 Kyungheedae-ro, Dongdaemun-gu, Seoul 02447, Korea

Tel: +82-2-958-8474, Fax: +82-2-958-8470, E-mail: happyjy416@gmail.com 
of the patients with Non-AR according to the conventional classification scheme were diagnosed with LAR when the above diagnostic criteria were applied. ${ }^{611) 12)}$

Even though there are many reports evaluating the relationship between lower respiratory tract diseases such as asthma and sinonasal diseases including CRS in patients with $\mathrm{AR}$, the role of allergic reaction in nasal mucosa on lower or upper airway diseases has not been identified well. Interestingly, previous studies have reported that allergic symptoms are associated with increases of local production of tryptase and ECP that is known as a poor prognostic factor of CRS. ${ }^{10)}$ In this study, we aimed to evaluate the association of patient-reported local allergic symptoms (LAS) such as sneezing and itching with the recurrence of CRS in AR and Non-AR patients.

\section{MATERIALS AND METHODS}

\section{Patients}

The medical records of patients with CRS who were admitted to Kyung Hee University Hospital between July 2014 and June 2015 and underwent endoscopic sinus surgery were reviewed. Patients who were under 18 years of age or who had asthma, diabetes mellitus, immunodeficiency, fungal sinusitis, or odontogenic sinusitis were excluded from the study. An AST, Multiple Allergosorbent Test (MAST), total IgE measurement, endoscopy, and paranasal sinus computed tomography (PNS CT) were performed prior to surgery. The study was approved by the KHMC Institutional Review Board (No. 2015-07-410).

\section{Diagnosis of CRS}

Patients were diagnosed with CRS if they suffered from symptoms such as congestion, nasal obstruction, facial pain, or hyposmia / anosmia for greater than 12 weeks, regardless of whether antibiotics were prescribed. ${ }^{113)}$ Evaluation of the nasal cavity was performed using an endoscope to confirm any findings including purulent mucus or edema in the middle meatus or anterior ethmoid region, or polyps in nasal cavity. Patients were diagnosed with CRS when opacity or thickening of the sinus mucosa was noted on PNS CT. ${ }^{\left.1{ }^{2}\right)}$ Endoscopic sinus surgery was performed on patients who did not respond to appropriate medication.

\section{Recurrence of CRS}

We evaluated the rate of recurrence of CRS in patients who underwent endoscopic sinus surgery. Patients with recurrent symptoms, mucopurulent discharge, and mucosal edema noted on endoscopy, sinus opacification visible on PNS $\mathrm{CT}$, or patients who did not respond to medication or underwent revision surgery were considered to have recurrent CRS. ${ }^{13) 14)}$

\section{Diagnosis of AR}

The diagnosis of AR was based on a history of allergic symptoms and diagnostic results consistent with the Allergic Rhinitis and its Impact on Asthma (ARIA) guidelines. ${ }^{15)}$ Allergic symptoms included rhinorrhea, sneezing, nasal obstruction, and itching. Diagnostic results included a positive AST or MAST indicating the presence of sIgE antibodies to aeroallergens. AST was performed with the most prevalent allergens, including Dermatophagoides pteronyssinus, Dermatophagoides farinae, Aspergillus, Alternaria, cockroach, tree, grass, weed, dog and cat. Histamine (10 mg/ $\mathrm{mL}$ ) and saline were used as positive and negative controls, respectively. A positive AST response was defined as a wheal diameter of $3 \mathrm{~mm}$ or greater or greater than a diameter of positive control. MAST was carried out with the allergens described above and food allergens. To be diagnosed with AR, a patient required more than three positive AST results and two positive MAST results. ${ }^{16)}$

\section{Assessment of symptoms}

Total nasal symptom scores (TNSS) were used to assess the severity of preoperative symptoms. We used a fourpoint scale $(0=$ no symptoms, $1=$ mild symptoms, $2=$ moderate symptoms, $3=$ severe symptoms) to grade four discrete symptoms: rhinorrhea, nasal obstruction, nasal itching, and sneezing. Total scores ranged from 0 (no symptoms) to 12 (maximum severity). ${ }^{17}$

\section{Local allergic symptoms (LAS)}

Among the four symptoms, ${ }^{18)}$ sneezing and nasal itching were categorized as LAS. Rhinorrhea and nasal obstruction were excluded from the categorization because they were also associated with CRS. The study population was categorized as either LAS $(+)$ or LAS $(-)$ group. LAS was determined positive if TNSS score included more than one point for nasal itching or sneezing.

\section{Statistical analyses}

All statistical analyses were performed using SPSS ver- 
sion 21.0 (SPSS Inc., Chicago, IL, USA). The $t$ tests and $\chi^{2}$ tests were used to describe the differences in terms of demographics and preoperative TNSS between each group. The $\chi^{2}$ tests was used to compare the rates of recurrence of CRS with LAS. Statistical significance was set at $95 \%$ or higher $(\mathrm{p}<0.05)$. The Institutional Review Board of Kyung Hee University Hospital approved this study.

\section{RESULTS}

\section{Patient demographics}

Of the 64 total patients, 27 belonged to the AR group and 37 to the Non-AR group. There was no significant difference in age, sex, and TNSS between the two groups ( $p>0.05$ ). However, the rhinorrhea and sneezing scores were significantly higher in the AR group than in the Non-AR group ( $\mathrm{p}=$ $0.024, p=0.041$; respectively; Table 1). Of the 64 patients, 45 belonged to the LAS (+) group and 19 to the LAS (-) group. There was no significant difference in age, sex, nasal obstruction score, and rhinorrhea score between the two groups ( $p>0.05)$. However, the TNSS was significantly higher in the LAS $(+)$ group than in the LAS $(-)$ group as expected $(\mathrm{p}=0.03)$. Furthermore, similar trend has been shown when we compared LAS (+) group and LAS (-) group in patients without AR ( $p>0.05$; Table 1$)$.

\section{Recurrence rate of CRS according to the presence of AR}

There was no significant difference in terms of the recurrence rate of CRS between patients with AR and those without AR ( $29.6 \%$ vs. $43.2 \%$, p=0.269; Fig. 1$)$. We further assessed if there is any difference in the recurrence rate of CRS according to the AST results or MAST results, showing no significant difference as expected $(\mathrm{p}=0.358$ and $\mathrm{p}=0.551$, respectively; Fig. 1).

\section{Recurrence rate of CRS according to the presence of LAS}

Given that presence of AR did not influence on the recurrence rate of CRS, we evaluated the effect of LAS on the recurrence rate of CRS. Of the total patients, the recurrence rate of CRS was significantly higher in the LAS $(+)$ group than those in the LAS (-) group $(46.7 \%$ vs. $15.8 \%$, $\mathrm{p}=0.020$; Fig. 2A).

Furthermore, when we assessed the association of LAS and recurrence rate of CRS in patients without AR, there was a similar trend, showing that recurrence rate of CRS was significantly higher in the LAS (+) group (14 of 25, $56 \%)$ than in the LAS (-) group ( 2 of $12,16.7 \%)(\mathrm{p}=0.024$; Fig. 2B). However, we couldn't find any significant difference in terms of the recurrence rate of CRS between patients with LAS and those without LAS in patients with AR (data not shown).

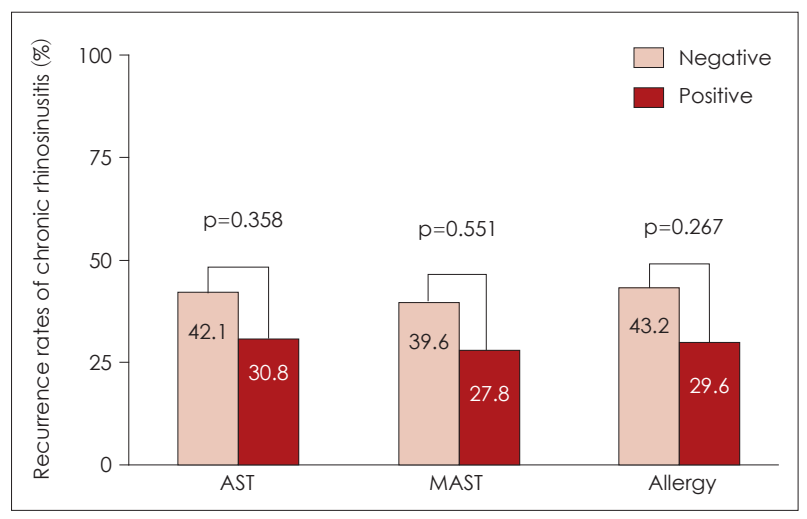

Fig. 1. Comparison of recurrence rates of chronic rhinosinusitis according to presence of allergy. AST: allergic skin prick test, MAST: Multiple Allergosorbent test, Allergy: AST positive response or MAST positive response.

Table 1. Demographics of patients

\begin{tabular}{|c|c|c|c|c|c|c|}
\hline \multirow{2}{*}{ Parameters } & \multicolumn{3}{|c|}{ Non-AR group $(n=37)$} & \multicolumn{3}{|c|}{ AR group $(n=27)$} \\
\hline & LAS (+) & LAS (-) & Total & LAS (+) & LAS (-) & Total \\
\hline Age (years) & $43.08 \pm 15.45$ & $45.50 \pm 14.34$ & $43.86 \pm 14.94$ & $40.70 \pm 18.04$ & $48.00 \pm 14.70$ & $42.59 \pm 17.27$ \\
\hline Sex ratio $(M / F)$ & $13 / 12$ & $6 / 6$ & $19 / 18$ & $4 / 8$ & $7 / 8$ & $11 / 16$ \\
\hline TNSS & $4.0 \pm 2.81$ & $2.83 \pm 2.75$ & $3.62 \pm 2.82$ & $6.0 \pm 2.59^{\dagger}$ & $2.53 \pm 1.73$ & $4.96 \pm 2.96$ \\
\hline Rhinorrhea score & $1.00 \pm 1.08$ & $1.16 \pm 1.26$ & $1.05 \pm 1.12$ & $1.60 \pm 0.94$ & $1.15 \pm 1.06$ & $1.51 \pm 0.89^{*}$ \\
\hline Nasal obstruction score & $1.40 \pm 1.04$ & $1.33 \pm 1.37$ & $1.37 \pm 1.13$ & $1.50 \pm 1.05$ & $1.24 \pm 1.01$ & $1.40 \pm 1.08$ \\
\hline Sneezing score & $0.88 \pm 0.66^{\dagger}$ & 0 & $0.70 \pm 0.77$ & $1.70 \pm 0.80^{\dagger}$ & 0 & $1.14 \pm 1.09 *$ \\
\hline Nasal itching score & $1.04 \pm 0.73^{\dagger}$ & 0 & $0.80 \pm 0.77$ & $1.50 \pm 0.76^{\dagger}$ & 0 & $1.03 \pm 0.97$ \\
\hline
\end{tabular}

$*: p<0.05$ when compared between AR and Non-AR, $\dagger: p<0.05$ when compared between LAS (+) and LAS (-). SD: standard deviation, TNSS: Total Nasal Symptoms Score, AR: allergic rhinitis, Non-AR: non-allergic rhinitis, LAS: local allergic symptoms (sneezing, itching) 

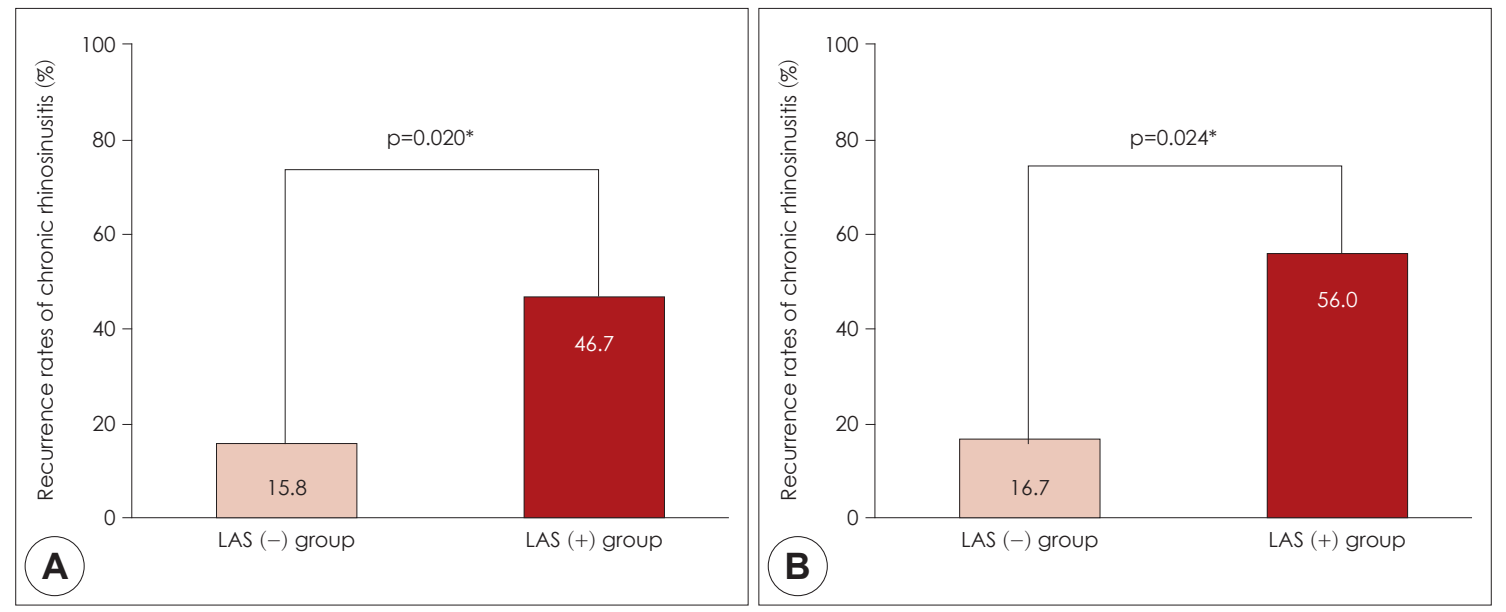

Fig. 2. Comparison of recurrence rates of chronic rhinosinusitis between LAS (+) group and LAS (-) group in all patients (A) and in nonAR patients (B). *: Statistically significant $(p<0.05)$. LAS: local allergic symptoms (sneezing, itching), Non-AR: non-allergic rhinitis.

\section{DISCUSSION}

In this study, we showed that the rate of recurrence of CRS was significantly higher in the LAS $(+)$ group than it was in the LAS (-) group. Furthermore, similar trend has been shown when we compared LAS (+) group and LAS (-) group in Non-AR patients.

Non-AR involves symptoms including sneezing, watery rhinorrhea, or having a congested nose without identified causes. Interestingly, recent studies have demonstrated an inflammatory infiltrate in the nasal mucosa of a subgroup of patients with non-AR that was very similar to that seen in patients with AR, suggesting the presence of LAR, or entopy, in these patients in the absence of systemic atopy. ${ }^{4) 5}$ Even where there is no evidence of the presence of atopy, local allergic responses are frequently observed. It has been demonstrated that local IgE in nasal tissues did not correlate with the presence of atopy as determined by positive skin prick test responses to inhalant allergens or serum IgE. ${ }^{19)}$ Furthermore, localized allergic responses to environmental allergens, microbial products, and fungal allergen in nasal mucosa have frequently shown regardless of the presence of atopy. ${ }^{20221)}$ However, it is not easy to diagnose LAR. The recommended diagnostic approach involves obtaining a detailed clinical history followed by AST. If a negative test result is obtained, serum total and SIgE antibody titers may be determined. If the sIgE antibody results are negative, the next step is to evaluate the target organ by quantifying nasal sIgE antibodies and performing an NAPT with the suspect allergen(s). ${ }^{71112(2) 22)}$ NAPT known to be the gold standard test for LAR, can be performed with either a single allergen (NAPT-S) or multiple allergens (NAPT-M) ${ }^{69911)}$ The major disadvantage of NAPT-S is that it requires a wash-out period prior to provocation with the four most common allergens in the surrounding environment (Dermatophagoides pteronyssinus, Alternaria alternata, Olea europea, and grass pollen), and as a result it takes one week for each antigen test. ${ }^{12)}$ The most important limitation is that it can be difficult to select the correct allergen for the provocation test if the patient has a negative response to the AST or serum sIgE test. Therefore, since the methods currently used to diagnose LAR are not efficient in terms of cost, time, and methodology, it may be necessary to replace these methods.

Rondon et al. used the visual analogue scale (VAS) to measure five symptoms (nasal obstruction, rhinorrhea, itching, sneezing, and ocular symptoms) when utilizing NAPT to diagnose LAR. ${ }^{10)}$ In the study, a significant association was observed between an increase in the nasal production of ECP and tryptase measured after NAPT and a worsening of nasal symptoms. ${ }^{10}$ A similar association was observed between an increase in nasal tryptase and a worsening of nasal sneezing and itching and a positive correlation was observed between an increase in ECP and worsening of nasal obstruction. ${ }^{10)}$ Separately, it has been reported that numerous inflammatory mediators including ECP, tryptase, and eotaxins are associated with prognostic outcomes of CRS after surgery and disease severity of CRS. ${ }^{2324)}$ Given that, development or worsening of nasal symptoms after exposure to allergens might be strongly related to allergic reaction with increased inflammatory mediators, resulting in poor prognosis of CRS. However, it has not been well iden- 
tified the association between symptoms of local allergic reaction and prognosis of CRS. Thus, in this study, we sought to identify if specific symptoms among LAS itself is associated with prognosis of CRS after surgery. Among the four major symptoms of AR, we included sneezing and itching and 2 other major symptoms including nasal obstruction and rhinorrhea were excluded from analysis because they were most likely to be associated with CRS. In this study, the rate of recurrence of CRS was significantly higher in the LAS (+) group than it was in the LAS (-) group, suggesting that control of the associated allergic symptoms might be able to contribute to reduce recurrence rate of CRS. Although allergy in part plays a role in sinonasal inflammatory diseases such as CRS, there is no difference in the incidence of CRS, especially CRSwNP between allergic versus non-allergic patients and discrepancy between IgE levels in serum and in NP tissue is frequently found, suggesting an independent localized allergic response that may play a critical role in sinonasal inflammation, particularly in CRS. ${ }^{25)}$ Supporting this, unlike LAS, we could not find any significantly different recurrence rate of CRS in AR versus NonAR groups. In addition, we only found that recurrence rate of CRS was higher in LAS (+) group compared to LAS (-) group in patients without AR. The reasons for the discrepancies are unclear, but possible hypotheses include that patients with AR were treated more aggressively for AR following surgery, suggesting that well-controlled immunologic reaction including allergic reaction in nasal mucosa might contribute to lower recurrence rate of CRS. However, to identify this hypothesis, future studies including enough number of patients with or without AR treatment are warranted. Additionally, since severity of AR might affect postoperative recurrence of CRS, further studies should also aim to assess the influence of AR severity for better understanding.

Studies evaluating the mechanism by which AR induces CRS have previously been performed. A typical pathologic mechanism is an IgE-mediated reaction involving accumulation of Th2 cells, lymphocytes, antigen-presenting cells (APC), eosinophils, macrophages, and mast cells in the mucosa of AR patients. ${ }^{5)}$ Immunoglobulin E is a local substance that can cause mast cells to become sensitized to common aeroallergens. ${ }^{3)}$ This process is known to cause nasal mucosal thickening, decreased ciliary function, and proliferation of goblet cells in the sinus, resulting in ventilatory and drainage disorders in the sinus. ${ }^{14)}$ In LAR patients, an in- flammatory response of the Th2 cells, production of sIgE to aeroallergen, an increase in inflammatory mediators, and an increase in ECP in the nasal mucosa were observed, which is similar to the mechanism noted to cause CRS in AR patients. ${ }^{5}$ In addition, swelling of the nasal mucosa may lead to reduced patency of the ostia, reduced transport capacity related to abnormalities of the cilia, and increased secretions in the sinus resulting in CRS. ${ }^{3)}$

The present study has the following limitations. First, we used LAS instead of performing NAPT in patients suspected of having LAR. Further studies are therefore required to determine whether LAS is representative of the symptoms present in patients with LAR. Second, because the sample size was small, additional prospective large-scale studies are required.

\section{CONCLUSION}

Regardless of the presence of AR, recurrence of CRS tends to be more common in patients with LAS including sneezing and itching. We suggest here that control of LAS might contribute to prognosis of CRS after surgery.

\section{Declaration of Interests}

The authors report no conflicts of interest. The authors alone are responsible for the content and writing of the paper.

\section{REFERENCES}

1) Rosenfeld RM, Piccirillo JF, Chandrasekhar SS, Brook I, Ashok Kumar K, Kramper M, et al. Clinical practice guideline (update): adult sinusitis. Otolaryngology-Head and Neck Surgery 2015;152(2_suppl):S1-39.

2) Pelikan Z, Pelikan-Filipek M. Role of nasal allergy in chronic maxillary sinusitis - diagnostic value of nasal challenge with allergen. Journal of allergy and clinical immunology 1990;86(4):484-91.

3) Baroody FM, Mucha SM, deTineo M, Naclerio RM. Evidence of maxillary sinus inflammation in seasonal allergic rhinitis. Otolaryngology--Head and Neck Surgery 2012;146(6):880-6.

4) Van Rijswijk J, Blom H, Fokkens W. Idiopathic rhinitis, the ongoing quest. Allergy 2005;60(12):1471-81.

5) Rondón C, Fernandez J, Canto G, Blanca M. Local allergic rhinitis: concept, clinical manifestations, and diagnostic approach. J Investig Allergol Clin Immunol 2010;20(5):364-71.

6) Rondón C, Doña I, Torres MJ, Campo P, Blanca M. Evolution of patients with nonallergic rhinitis supports conversion to allergic rhinitis. Journal of Allergy and Clinical Immunology 2009;123(5):1098102.

7) Arasi S, Pajno GB, Lau S, Matricardi PM. Local allergic rhinitis: a critical reappraisal from a paediatric perspective. Pediatric Allergy and Immunology 2016;27(6):569-73. 
8) Carney AS, Powe D, Huskisson R, Jones N. Atypical nasal challenges in patients with idiopathic rhinitis: more evidence for the existence of allergy in the absence of atopy? Clinical \& Experimental Allergy 2002;32(10):1436-40.

9) López S, Rondón C, Torres M, Campo P, Canto G, Fernandez R, et al. Immediate and dual response to nasal challenge with Dermatophagoides pteronyssinus in local allergic rhinitis. Clinical \& Experimental Allergy 2010;40(7):1007-14.

10) Rondón C, Fernández J, López S, Campo P, Doña I, Torres MJ, et al. Nasal inflammatory mediators and specific IgE production after nasal challenge with grass pollen in local allergic rhinitis. Journal of Allergy and Clinical Immunology 2009;124(5):1005-11. e1.

11) Rondón C, Canto G, Blanca M. Local allergic rhinitis: a new entity, characterization and further studies. Current Opinion in Allergy and Clinical Immunology 2010;10(1):1-7.

12) Rondón C, Campo P, Herrera R, Blanca-Lopez N, Melendez L, Canto $\mathrm{G}$, et al. Nasal allergen provocation test with multiple aeroallergens detects polysensitization in local allergic rhinitis. Journal of Allergy and Clinical Immunology 2011;128(6):1192-7.

13) Bhattacharyya T, Piccirillo J, Wippold FJ. Relationship between patient-based descriptions of sinusitis and paranasal sinus computed tomographic findings. Archives of Otolaryngology-Head \& Neck Surgery 1997;123(11):1189-92.

14) Benninger MS, Ferguson BJ, Hadley JA, Hamilos DL, Jacobs M, Kennedy DW, et al. Adult chronic rhinosinusitis: definitions, diagnosis, epidemiology, and pathophysiology. Otolaryngology-Head and Neck Surgery 2003;129(3):S1-S32.

15) Brożek JL, Bousquet J, Baena-Cagnani CE, Bonini S, Canonica GW, Casale TB, et al. Allergic Rhinitis and its Impact on Asthma (ARIA) guidelines: 2010 revision. Journal of Allergy and Clinical Immunology 2010;126(3):466-76.

16) Skoner DP. Allergic rhinitis: definition, epidemiology, pathophysi- ology, detection, and diagnosis. Journal of Allergy and Clinical Immunology 2001;108(1):S2-8.

17) Graft D, Aaronson D, Chervinsky P, Kaiser H, Melamed J, Pedinoff A, et al. A placebo-and active-controlled randomized trial of prophylactic treatment of seasonal allergic rhinitis with mometasone furoate aqueous nasal spray. Journal of Allergy and Clinical Immunology 1996;98(4):724-31.

18) Campo P, Eguiluz-Gracia I, Bogas G, Salas M, Plaza Seron C, Perez N, et al. Local allergic rhinitis: Implications for management. Clin Exp Allergy 2019;49:6-16.

19) Bachert C, Gevaert P, Holtappels G, Johansson S, Van Cauwenberge P. Total and specific IgE in nasal polyps is related to local eosinophilic inflammation. Journal of Allergy and Clinical Immunology 2001;107(4):607-14.

20) Powe D, Bonnin A, Jones N. 'Entopy': local allergy paradigm. Clinical \& Experimental Allergy 2010;40(7):987-97.

21) Sabirov A, Hamilton RG, Jacobs JB, Hillman DE, Lebowitz RA, Watts JD. Role of local immunoglobulin E specific for Alternaria alternata in the pathogenesis of nasal polyposis. The Laryngoscope 2008;118(1):4-9.

22) Settipane RA, editor Rhinitis: a dose of epidemiological reality. Allergy and asthma proceedings;2003: OceanSide Publications.

23) Lam M, Hull L, McLachlan R, Snidvongs K, Chin D, Pratt E, et al., editors. Clinical severity and epithelial endotypes in chronic rhinosinusitis. International forum of allergy \& rhinology;2013: Wiley Online Library.

24) De EC, Baroni S, Romitelli F, Luca L, Di WN, Passali GC, et al. Nasal lavage CCL24 levels correlate with eosinophils trafficking and symptoms in chronic sino-nasal eosinophilic inflammation. Rhinology 2011;49(2):174-9.

25) Caplin I, Haynes J, Spahn J. Are nasal polyps an allergic phenomenon? Annals of Allergy 1971;29(12):631-4. 